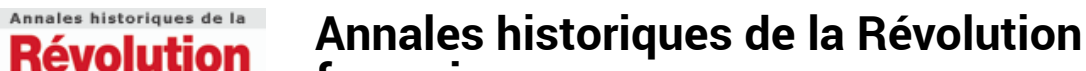 \\ française française
}

324 | avril-juin 2001

Louis Charles Antoine Desaix. Officier du roi, Général de la République

\section{Vertu antique et nouveaux héros}

La presse autour de la mort de Desaix et d'une bataille légendaire

\section{Raymonde Monnier}

\section{(2) OpenEdition \\ Journals}

Édition électronique

URL : https://journals.openedition.org/ahrf/399

DOI : 10.4000/ahrf.399

ISSN : 1952-403X

Éditeur :

Armand Colin, Société des études robespierristes

Édition imprimée

Date de publication : 1 juin 2001

Pagination : 113-125

ISSN : 0003-4436

Référence électronique

Raymonde Monnier, "Vertu antique et nouveaux héros », Annales historiques de la Révolution française [En ligne], 324 | avril-juin 2001, mis en ligne le 22 mai 2006, consulté le 24 avril 2022. URL : http:// journals.openedition.org/ahrf/399 ; DOI : https://doi.org/10.4000/ahrf.399

Ce document a été généré automatiquement le 24 avril 2022.

Tous droits réservés 


\title{
Vertu antique et nouveaux héros
}

\author{
La presse autour de la mort de Desaix et d'une bataille légendaire
}

\author{
Raymonde Monnier
}

\section{C'était un caractère tout à fait antique}

(Mémorial de Sainte-Hélène)

1 Cette recherche sur la mort du général Desaix s'inscrit dans un travail plus large sur la notion de patrie et le culte de la patrie sous la Révolution. Le moment de Marengo est intéressant pour étudier l'esprit public et les mutations en cours dans les thèmes et les formes du culte des héros. D'autres traitent ici des monuments et des éloges officiels ; je limiterai mon propos aux hommages particuliers et aux manifestations de l'opinion, à partir des discours imprimés et des célébrations dont il est rendu compte dans la presse parisienne. Celle-ci a été singulièrement réduite depuis l'arrêté du 27 nivôse an VIII : on ne compte plus qu'une douzaine de journaux mais ils n'en constituent pas moins une source appréciable, car l'absence de liberté politique réelle incite les rédacteurs à se tourner vers la sphère culturelle. L'intérêt de la recherche dépasse les circonstances et les hommages rendus à un jeune général de la République, car la période est celle de déplacements caractéristiques dans la célébration des héros et la constitution du Panthéon national.

2 Jean-Claude Bonnet a montré comment la résurgence du culte des grands hommes après 1750 a contribué à construire, à partir des éloges académiques, une sorte de Panthéon de papier avant même la consécration de l'édifice par la Révolution'1. S'il y a dans les célébrations de la Révolution réemploi de tout un passé de formes sacrées ou profanes, on peut se demander dans quelle mesure les cultes révolutionnaires se situent dans la continuité de cette tradition, qui annonce l'avènement des mérites et des talents, mais qui réservait ses éloges aux hommes éminents du passé plus qu'aux héros, et dont la figure centrale était celle de la monarchie. Sous la Révolution, le culte des grands hommes quitte les lieux clos de l'Académie et des salons pour se déployer dans la Cité, témoignant de l'avènement d'une autre légitimité symbolique et de l'évolution radicale vers de nouvelles valeurs fondées sur le mérite et la vertu civiques. Le temple ouvert des figures promises à l'héroïsation avec les martyrs de la Liberté, les avatars de la mémoire immédiate qu'illustre au Panthéon le chassé-croisé des entrants 
et des sortants, les vicissitudes au xixe siècle d'un édifice qui ne retrouvera sa vocation révolutionnaire qu'à la mort de Hugo, montrent l'importance que revêt dans les débats et les combats de la République l'enjeu de la mémoire nationale.

C'est aussi depuis 1885 qu'est durablement conservée l'inscription que la Révolution avait placée au fronton du temple de la Renommée: Aux grands hommes, la Patrie reconnaissante. La notion de patrie ${ }^{2}$, qui s'est développée au xviiie siècle dans les termes $\mathrm{du}$ républicanisme classique, est devenue le référent d'une instance légitime supérieure, une valeur nécessaire au vivre ensemble, qui est aussi une espérance de dépassement et de plénitude de soi dans une vraie communauté. Chez Rousseau, l'exemple de Rome, cité de l'amour exclusif de la patrie, inspire l'idéal de la Cité patrie, lieu d'intégration harmonieuse des citoyens, où l'individu éprouve le sentiment de sa liberté dans la patrie qu'il aime ${ }^{3}$. Sous la Révolution, la notion prend dans les événements une valeur affective si forte que le nom lui-même devient une force vivante et agissante, dont on suit les effets et l'évolution dans les discours et les manifestations publiques.

4 La crise de l'été 1792 donne au mot patrie une intensité sans précédent et à la notion une puissance extrême de contenu et d'expression ; l'enthousiasme est le moteur de l'élan collectif qui mène en 1793 à ce qu'il n'est pas excessif d'appeler un véritable culte de la Patrie, où les célébrations jouent un rôle médiateur entre culture populaire et culture politique des élites révolutionnaires ${ }^{4}$. La notion est au centre des déterminations sociales, politiques, morales et religieuses mises en œuvre pour préserver l'unité révolutionnaire quand la situation exige la cohésion nationale la plus forte. Le cycle de fêtes où la trinité patriotique Marat-Lepeletier-Chalier est associée à d'autres martyrs, aux philosophes et aux héros de l'Antiquité, suscite une relance de l'énergie révolutionnaire analogue au mot d'ordre de la levée en masse: Le peuple français debout contre les tyrans. L'héroïsation publique de la figure virile de Brutus, modèle du devoir républicain, fait partie du nouveau système de représentations $\mathrm{du}$ peuple "régénéré ", imposant sur le thème de la terreur et de la vertu, l'exemple du dévouement inflexible à la patrie ${ }^{5}$.

5 Le culte des martyrs, dans ses hommages au patriotisme militant prépare l'exaltation des défenseurs de la patrie anonymes, des actions collectives et individuelles célébrées dans les Annales du civisme et de la vertu. Dans son rapport sur les fêtes nationales, Robespierre développe les principes du nouveau Panthéon républicain : «Nos sublimes voisins entretiennent gravement l'univers de la santé du roi, de ses divertissements, de ses voyages [...] Nous lui apprendrons, nous, les noms et les vertus des héros morts en combattant pour la liberté [...] Combien de noms dignes d'être inscrits dans les fastes de l'histoire, demeurent ensevelis dans l'obscurité! Mânes inconnus et révérés, si vous échappez à la célébrité, vous n'échapperez point à notre tendre reconnoissance ${ }^{6}$.

6 La Révolution égalitaire de 1793 préfère à la gloire des grands hommes la vertu et le sacrifice des humbles, et célèbre la bravoure et l'héroïsme au quotidien dans les armées de la République ${ }^{7}$. Le développement du Panthéon révolutionnaire est indissociable d'un contexte culturel et politique qui a donné vie au forum dans un souci d'intégration collective et de reconnaissance réciproque dans les valeurs de la République. L'éloquence vivante du patriotisme en acte remplace à l'Assemblée nationale et dans la Cité le style onctueux de l'éloge à l'Académie, pour électriser les âmes et cimenter les énergies dans la défense de la patrie. 
7 Par la suite, les hommages aux actions héroïques et aux armées, tout en continuant à faire référence aux Anciens et à honorer la bravoure des défenseurs de la patrie, distinguent particulièrement l'illustre figure de leurs chefs pour inscrire leur vertu dans le marbre. Les fêtes séparées ne disparaissent pas sous le Directoire, mais elles quittent le forum pour se tenir au sein d'institutions particulières. Les fêtes nationales gardent un certain éclat; elles réservent une place de choix aux démonstrations militaires, tentent de susciter l'intérêt public par des jeux au Champ-de-Mars, sans grand succès au dire des journaux. La République poursuit son dialogue avec les ombres dans des cérémonies funèbres en l'honneur des jeunes généraux morts à son service : Marceau et Hoche en l'an VI, Joubert en l'an VII, Desaix et Kléber en l'an VIII. L'hommage de la Patrie aux héros d'une épopée en marche a supplanté sur la scène nationale les gloires évanescentes des distinctions académiques.

Hommages officiels et célébrations séparées

8 Le moment de Marengo permet, comme celui de l'automne 1793, de saisir le sens de la dialectique entre fêtes nationales et célébrations séparées, entre espace public et médium des institutions dans l'expression de l'esprit public, et le rôle du culte des héros dans le ralliement aux valeurs du régime. Le Consulat introduit une rupture avec le Directoire dans la politique des fêtes, qui n'est pas sans lien avec l'attitude vis-à-vis de la religion : il abandonne les fêtes décadaires pour ne conserver que deux grandes fêtes nationales, l'anniversaire du 14 juillet 1789, et celui de la fondation de la République, le $1 \mathrm{er}$ vendémiaire; mais parallèlement on assiste à un regain des célébrations séparées dans les institutions particulières, en pleine renaissance. La victoire de Marengo, la mort des généraux Desaix et Kléber, le retour du Premier consul à Paris sont, pour les élites dans les institutions et pour le peuple dans des fêtes nationales, autant d'occasions de manifester et d'exprimer leur adhésion active au régime et aux valeurs qu'il représente.

9 Dans les trois derniers mois de l'an VIII, de l'annonce de la victoire de Marengo à la fête de la République, ont lieu à Paris plusieurs cérémonies officielles au Temple et au Champ de Mars, dont la fête anniversaire du 14 juillet. La presse rend compte ou signale dans la même période, à côté de services religieux pour les héros morts à Marengo ${ }^{8}$, au moins une douzaine de célébrations civiles séparées, dans des temples ou des institutions particulières, dont moitié du 10 au 21 messidor: après une fête en l'honneur de Rousseau le 10 au Temple de la Victoire (Saint-Sulpice), on célèbre les victoires des armées le 15 au Temple du Commerce (Saint-Méry) ${ }^{9}$. Deux séances extraordinaires du Portique républicain, les 16 et 21 messidor, sont consacrées aux triomphes de la République et à la mémoire de Desaix et de ses compagnons d'armes ; un hommage leur est encore rendu le 20 à l'Institut national. La clôture des cours du Lycée républicain le 29 est l'occasion de nouveaux éloges. Les journaux signalent encore l'hommage rendu à la séance publique de la Société philotechnique le 20 thermidor, puis le 29 fructidor. L'éloge du général Desaix par Joseph Lavallée a été publié $^{10}$, de même que quelques autres, prononcés à la même époque: son oraison funèbre par Destravault, publiée en l'an VIII et son éloge funèbre par Despréaux, prononcé à l'Institution oratoire et dramatique, les 5 et 10 vendémiaire an IX.

Il y eut d'autres hommages littéraires, des pièces, des poèmes, des odes, des chants, dont la presse publie des extraits. Dès l'annonce de sa mort le Journal de Paris consacre une notice à Desaix: "il étoit d'une simplicité de caractère admirable. Il aimoit la guerre comme art, il s'échauffoit en racontant les actions où il s'étoit trouvé. Ses yeux 
alors devenoient étincelans de génie. Ceux qui conversoient avec lui familièrement, éprouvoient une surprise agréable, en l'entendant passer subitement du récit d'une bataille, à des sujets d'histoire naturelle ». Les dernières paroles qu'on lui attribue signalent « un caractère sans tache, une bravoure réunie à tous les dons du génie " ${ }^{11}$. Les dépêches officielles relatant la victoire de Marengo sont aussitôt publiées dans la presse, tant le rapport de Berthier que le Bulletin de l'armée de réserve, qui était à peu de chose près assez conforme à la réalité. Donc dès le 3 messidor, Paris sait que l'intervention de Desaix a retourné le sort de la bataille. On vante la bravoure des grenadiers de la garde consulaire; quelques journaux donnent aussi des témoignages du caractère sanglant des combats : l'horreur du carnage a ébranlé ceux qui s'y sont trouvés ${ }^{12}$.

11 Paris n'en manifeste pas moins un grand enthousiasme à l'annonce d'une victoire qui tient du prodige et une admiration unanime à Bonaparte et à l'armée. La presse célèbre l'événement, ainsi La Décade du 10 messidor : «Quand nous présagions dans le dernier Numéro, des succès en Italie, nous ne pouvions penser que dix jours après, nous en devrions annoncer de si éclatants qu'ils tiennent du prodige [...] De quoi nous serviraitil de raconter aujourd'hui en détail des faits qui ont parcouru le monde avec la rapidité de l'éclair, qui sont déjà dans les bouches des enfans et des femmes [sic] ».

Éloges à la vertu héroïque

12 Les auteurs d'éloges funèbres dans les institutions particulières sont pour la plupart des hommes de lettres, qui connaissent à l'évidence les canons du genre et l'art de l'éloquence: Lavallée est journaliste, auteur d'ouvrages littéraires et historiques, membre de plusieurs sociétés savantes, Simien Despréaux est professeur de BellesLettres ; Joseph-Xavier Destravault, lui, est homme de loi et dédie son éloge à un ancien constituant, juge au tribunal de Cassation, Claude-Alexis Cochard ${ }^{13}$. Les deux grandes parties de son éloge de Desaix retracent la brillante carrière d'un général dont la courte vie fut tout entière placée sous le signe des Lumières et de la vertu: à la valeur héroïque et au génie du général républicain dans la guerre, répond la noble ambition d'aller "nous conquérir des amis, par-delà les mers». C'est en égypte qu'il peut déployer ses rares qualités dans une harmonie réciproque avec ses soldats, et que son grand caractère lui vaut le titre de sultan juste : «Jamais les vertus, la gloire militaire, n'ont été si éclatantes ; mais aussi jamais la cause qui les anima, ne fut plus grande, plus généreuse ». L'illusion de la paix croise le mythe de l'expédition civilisatrice. La mort héroïque de Desaix - évoquée au présent - donne sens à ses regrets de n'avoir pas fait assez pour la patrie : elle a devancé «cette révolution, la seule désirable, la seule à laquelle nous pouvons tous coopérer efficacement, sans verser le sang : celle du goût et des mœurs ".

13 L'éloge de Joseph Lavallée est un morceau d'éloquence héroïque, qui est par ailleurs salué dans le Journal des Arts, comme un éloge "plein d'âme, de sentiment et de vérité $»^{14}$. Lavallée est un ancien militaire et écrit dans plusieurs journaux, dont le Journal des Défenseurs de la patrie, dont il est le principal rédacteur. Son style est persuasif et entraînant comme les tranches de vie qu'il évoque, de l'enfance de Desaix à ses années de collège à Effiat. C'est là que «sage avant l'âge de la sagesse», il s'initie aux sciences, prend goût à l'histoire naturelle; mais c'est dans l'armée de la République qu'on le voit «transformer en grandes actions ce qu'il n'avait encore qu'en grandes idées ". 

cœur de chaque soldat, à la bravoure naturelle des Français, une ardeur de dangers qui ressemble presque à la soif de la vengeance ", Lavallée suit son héros dans les campagnes de l'armée du Rhin. Le morceau de bravoure est le passage du Rhin, l'un des plus grands moments de "l'intrépidité guerrière ", où l'armée réussit un exploit qui tient du prodige. L'orateur peint le tumulte des hommes et des éléments, «le ciel, complice de ce spectacle formidable, unissant les orages de l'air aux orages des passions [...] et du fleuve, et des Césars, et des élémens; l'armée passe : elle est passée [...] la liberté triomphe ; et la fortune de l'an V s'assied sur l'immortalité ».

Avec l'expédition d'égypte, terre de légende, où tout est colossal même les vices, le destin de Desaix rencontre le génie de Bonaparte, pour porter dans ce «foyer des passions de l'espèce " le "génie de la raison ». L'éloge du Premier consul se mêle à l'éloge du général dans l'évocation mythique de l'expédition et de la dernière campagne de l'armée de réserve. Signe des espérances nées de Marengo, l'épilogue de cet éloge de l'héroïsme guerrier se plaît à imaginer la vie qu'eût menée Desaix dans la paix, s'occupant de sa mère et cultivant sa terre, rendu ainsi à la vie champêtre qu'il aimait.

La prestation de Despreaux, tranche par le ton sinon par la forme avec les précédentes ${ }^{15}$. C'est un hommage appuyé au Premier consul à qui l'éloge est dédié ; en même temps les commentaires sur la vie de Desaix sont ceux d'un nostalgique de l'Ancien Régime. L'auteur regrette d'avoir peu de détails sur son caractère car «c'est dans le particulier, c'est dans le calme de la vie privée qu'on se montre tel qu'on est, sans apprêt et sans déguisement ». On peut se demander si l'absence de faits importe vraiment à ce professeur de Belles-Lettres, qui connaît les règles de l'art et les grands canons du genre, de Thomas à La Harpe, dans la distinction du mérite et de la vertu. Tout en disant céder à une exigence de vérité, son éloge du jeune général républicain propose un parallèle surprenant avec celui du maréchal Catinat ${ }^{16}$.

17 Ainsi dépouillé des vertus guerrières généralement attribuées aux grands capitaines, Desaix est loué pour ses vertus sociales. «Comme homme privé, Desaix est un modèle de douceur, de bonté, d'amitié, de piété filiale. Comme homme public et guerrier, Desaix est un modèle de désintéressement, de conduite, de courage et d'intrépidité ». Ainsi résumées, les deux parties de l'éloge développent les qualités morales, puis les actions courageuses d'un officier philosophe, qui «n'envisage la guerre que comme un acheminement à la paix ». Aimant l'étude, la littérature et les arts, il emploie "sa philosophie et sa prudence à régler sa conduite, à embellir son âme ", loin des amusements frivoles qui perdent la jeunesse. Les circonstances de sa vie offrent l'exemple de sa grande simplicité et de sa modestie, "vertu sublime », de sa frugalité spartiate. Désintéressé jusqu'au stoïcisme, cet "homme libéral et compatissant» pratique "l'humanité, la bienfaisance, la pitié pour les malheureux", toutes vertus morales qu'il n'abandonne pas dans les camps.

Le récit de sa vie guerrière relate ses traits de courage, sa prudence et sa sollicitude envers ses soldats, qui lui valent l'estime et la confiance de la troupe; le passage du Rhin est d'une inspiration plus lyrique pour faire l'éloge de l'armée tout entière : « Ce ne sont point des hommes qui s'avancent, ce sont des héros, que dis-je? ce sont des dieux de la guerre ». Après l'évocation de l'expédition d'égypte, toute à la gloire de Bonaparte, vient celle du champ de bataille de Marengo, spectacle épouvantable, «immense tombeau»! La péroraison clôt l'éloge sur l'espoir qu'avec le siècle se

Annales historiques de la Révolution française, 324 | avril-juin 2001 
perdent dans l'oubli les derniers vestiges des plaies que les guerres ont faites à l'humanité. Il est consolant de porter ses regards sur un jeune héros «qui marche à regret sur les trophées de la victoire».

19 Ces éloges de Desaix, on le voit, sont assez différents quand à l'inspiration ; mais au moment où on envisage de faire renaître l'Académie de ses cendres, on note un retour aux codes classiques du genre, à commencer par le plan en deux divisions entre exorde et péroraison. Les circonstances de la Révolution font qu'ils obéissent sans difficulté au canon qui veut que le héros ait été persécuté. Les effets des lois de suspicion sont différemment appréciées, mais la captivité infligée aux Français par l'amiral Keith s'intègre naturellement dans le récit de son retour d'égypte. La mort héroïque est tout entière rapportée à une vie exemplaire, où le cadre et les détails les plus contingents de son enfance et de sa jeunesse sont évoqués pour prouver que ses vertus « ont été aussi pures que sublimes » (Despreaux) : entouré dès la naissance « des grands spectacles de la nature, et des grands souvenirs de l'histoire " (Lavallée), dans un pays où règnent la simplicité et «la franche austérité des mœurs " (Destravault), il trouve à Effiat, après l'éducation de sa famille, « la richesse de l'éducation nationale » : « ce fut là qu'Homère lui fit aimer l'héroïsme, Tacite la liberté, Fénelon la vertu» (Lavallée).

On prête à Desaix l'ardeur sublime du héros qui ne rêve de gloire que pour s'égaler aux héros de Plutarque et vivre dans la mémoire de la patrie. L'amour de la gloire est le ressort de ses passions, « mais dans son cœur, il l'épure, il l'enchaîne, il le comprime, il l'exhale à son gré ; il ne s'en sert que pour illustrer son pays » (Destravault). C'est ce qu'exprimait déjà Daunou au Tribunat le 3 messidor: "Qu'on ne demande plus comment tous ces héros ont acquis, si jeunes, tant de gloire; Desaix en mourant a révélé leur secret. C'est l'amour de la gloire qui les guide. Ce sentiment s'épure dans les Républiques [...] il leur fait faire tout ce qu'il est possible de faire pour vivre dans la postérité $»^{17}$.

21 Comme les Spartiates, qui regardaient la modestie et la simplicité comme la source de leur valeur, le héros à l'antique est ennemi de l'ostentation. Desaix à qui la nature avait prodigué les qualités les plus aimables, demeurait d'une rare simplicité d'habit : «Un surtout bleu formait son vêtement ordinaire, et il ne portait le grand uniforme de son grade qu'aux jours de bataille » (Despreaux). Ses dernières paroles, même révoquées en doute, invitent à l'éloge et en appellent au respect de la postérité pour un tel héroïsme ; les auteurs en font un élément du discours; placées en exergue, elles résument les vertus sublimes du héros. Elles révèlent à quel degré de modestie Desaix «soutenait l'éclat de ses vertus ", modestie sublime qui, pour Lavallée, passe ce que l'on connaît de l'étude des «belles âmes ». Le refus des distinctions et des gratifications personnelles s'intègre dans l'éloge du guerrier, tout entier tourné vers le sacrifice à la patrie. Par delà le respect dû aux règles du genre, les éloges ont intégré les valeurs de l'héroïsme révolutionnaire.

Ce qui est mis en avant et proposé à la sensibilité de l'auditoire, ce n'est pas l'exploit individuel, mais l'héroïsme collectif d'une grande aventure libératrice. Il est des combats dont la gloire revient à l'armée entière, et qui prennent dans l'instant valeur d'épopée. C'était le cas de ceux livrés par l'armée de Rhin et Moselle sur le fleuve frontière. Moreau écrivait déjà, dans son rapport sur le passage du Rhin : «Il est inutile de faire d'éloges particuliers, après le récit des combats où il a fallu déployer autant de courage, il suffit de savoir qu'on y a pris part $»^{18}$. «Glorifier le général, dit Destravault, c'est assez annoncer combien l'armée est digne de l'être ». L'évocation sensible des 
exploits sur le Rhin se mêlait à l'hommage rendu à la glorieuse campagne de l'armée de réserve et à ses succès immédiats en Italie.

Le décor et le rituel demeurent ceux des cultes de la patrie, où l'inspiration religieuse se mêle aux motifs antiques et patriotiques, de la mise en scène funèbre du Tribunat à la célébration du Portique républicain, le 16 messidor, annoncée par le Journal des Hommes Libres comme le Te Deum des républicains ${ }^{19}$. Au milieu d'un grand appareil militaire, les fêtes officielles offrent les thèmes d'une nouvelle filiation héroïque : le 1er vendémiaire, Bonaparte pose la première pierre du monument place des Victoires, où " étoit élevé un temple antique en granit, orné des bustes des généraux Kléber et Desaix $»^{20}$. Il s'agissait en fait d'une maquette en vraie grandeur du projet de Chalgrin, inspiré du temple de Denderah, d'après le dessin de Denon ${ }^{21}$. Tous les arts, l'éloquence, la poésie et la musique, participent au succès de ces célébrations, ainsi à la Société philotechnique, où la séance publique se termine par une scène des chants d'Ossian, chantée par Bertin ${ }^{22}$. Le tableau de Girodet-Trioson, l'« Apothéose des héros français morts pour la patrie", est caractéristique de cette quête de nouvelles racines nationales.

L'hommage à une armée de héros

Les cérémonies particulières dont il est rendu compte dans la presse se mêlent en messidor, au tribut d'admiration et de reconnaissance qu'offre Paris à «l'armée de héros » et au général-consul. La campagne de l'an VIII s'y prête d'autant mieux qu'elle est jalonnée d'exploits qui prennent tout de suite une dimension légendaire. Un officier écrivait déjà le 4 prairial de la traversée des Alpes par l'armée de Bonaparte : "Cette marche est admirable, l'histoire la racontera et nos descendants y croiront à peine ${ }^{23}$. Dans ses commentaires des événements, la presse autorisée, toutes tendances confondues, use plus de son pouvoir d'amplification pour créer un consensus autour du nouveau régime que d'une véritable réflexion critique. D'autant que si on exprime à l'occasion des réserves sur l'orientation culturelle ou religieuse, ou si on souligne les tendances partisanes d'un autre journal, l'opinion générale est à la dénonciation des partis qui font obstacle à l'unité nationale : " pour assurer la paix de l'avenir, il faut, une bonne fois, le dégager du passé»". Apparaissant comme "étranger à tous les systèmes", Bonaparte semble porter tous les espoirs de la République dans une "glorieuse pacification ». La Décade adopte en thermidor un ton plus réservé : «C'est pour avoir la paix, pour en obtenir une juste et honorable, pour réprimer l'ambition, et sur-tout pour garantir son indépendance, qu'il est permis de soutenir la guerre $»^{25}$. Mais en messidor, l'heure est à la confiance et à la joie universelle.

Dès l'annonce de la victoire, connue à Paris le 2 messidor, les fêtes spontanées se succèdent pour fêter Marengo, puis le retour du Premier consul, avant l'apothéose patriotique du 14 juillet. La presse décrit l'allégresse qui accueille la nouvelle : « Jamais, depuis 1789, la joie ne s'était manifestée dans Paris avec plus de franchise et un caractère de patriotisme plus touchant [...] On sut bientôt qu'il s'agissait de victoires importantes [...] Les salves d'artillerie se prolongeaient, on affichait dans les rues le bulletin de l'armée de réserve ${ }^{26}$. La population, comme "frappée d'une étincelle électrique ", est curieuse de tout savoir, de tout entendre. Le soir, Paris est illuminé : «C'est la première illumination spontanée qui ait eu lieu depuis 9 ans. L'allégresse est générale. Tout le jour le travail a été suspendu ». Le lendemain dimanche les théâtres retentissent $«$ de chants de victoire et de cris d'allégresse $»^{27}$. 
26 Au retour de Bonaparte, le 13 messidor, les Parisiens sortent à nouveau dans les rues pour « fêter l'héroïsme ». " Une foule immense remplissoit la terrasse et les cours des Tuileries. Jamais on n'avoit vu plus universellement briller sur les visages un air de joie, de contentement et de reconnoissance $»^{28}$. « Nul programme, nulle invitation, nul ordre n'avaient indiqué les dispositions de cette fête ; et cependant il n'en fut point de plus aimable ni de plus touchante. La franchise avait tout prévu, le sentiment a tout exécuté, le cœur a tout animé [...] La soirée était superbe. À minuit la foule était encore immense dans toutes les rues. [...] Point de danses, point de musique [...] mais du calme, de l'épanchement, de la cordialité, de la fraternité ; les douces jouissances enfin d'un peuple libre... $»^{29}$. «Chacun croit tenir la paix, et nous pouvons assurer que cette illusion flatteuse ne tardera pas à se réaliser". On note la même "satisfaction générale » au faubourg Antoine ${ }^{30}$.

27 Cet enthousiasme mène à la grande réussite de la fête de la Concorde le 25 messidor, où les récits circonstanciés des journaux montrent que Bonaparte a véritablement conquis Paris. Tout concourrait à donner un nouvel essor au sentiment patriotique : la fête de l'Union des Français, décidée pour célébrer la pacification de l'Ouest, avait été reportée au 14 juillet, jour où devaient également être posées les premières pierres des colonnes départementales, et de la colonne nationale de la place de la Concorde pour «que partout, à la même heure, retentissent les noms de ces généreux guerriers dont le sang a coulé pour la patrie $»^{31}$. L'empressement des Parisiens à se porter «en foule aux divers lieux de la cérémonie " entraîne dans la presse un parallèle avec la commémoration du 14 juillet 1790, "si célèbre par la fédération générale »: "Il n'appartenait qu'à un gouvernement fondé sur la justice et la raison, de rappeler les douces émotions que fit naître cette explosion vraiment nationale, vers un nouvel ordre de choses, en consacrant ce jour à une fête qui, en rappelant cette grande époque, fût célébrée sous les auspices de la concorde » $^{32}$. «Paris n'a jamais offert un coup d'œil aussi étonnant ", de la cérémonie solennelle à la mémoire de Desaix au Temple de Mars au magnifique spectacle qu'offrait la place de la Concorde, « où tout élevait l'âme ; tout disait, voilà les Francs ». «Ce qui ne peut se décrire de cette journée, c'est l'affluence des citoyens par-tout où l'on pouvoit espérer de voir Bonaparte, c'est l'allégresse publique, la confiance, l'admiration, la gratitude que lui ont exprimées des acclamations non interrompues. Ceux qui l'avoient vu courroient pour gagner un passage où ils pussent le revoir encore ». Le Journal des Hommes libres évoque Bonaparte qui savoure son triomphe, et «tel le Germanicus de Tacite, écoutant les bénédictions de la nation, jouit, comme Germanicus, de sa réputation $»^{33}$. Au Champ-de-Mars, où on avait préparé des jeux, « lorsque le Premier consul eut passé la revue de la garnison, le peuple qui voulait voir de plus près le magistrat suprême, si digne de l'estime nationale, est descendu des talus, et s'est répandu dans l'enceinte où les jeux devaient s'exécuter. On a respecté, comme cela se doit chez un peuple libre, cet acte de sa volonté. En conséquence, les courses n'ont point eu lieu $»^{34}$.

29 Sous le Consulat, le sentiment patriotique ne s'exprime plus par le medium d'une sociabilité militante et acculturante ; l'adhésion populaire, même spontanée, fait partie du spectacle des célébrations nationales dont la figure centrale est celle du Premier consul. Le culte des héros dans les institutions permet de tester l'opinion des élites attachées ou résignées à l'œuvre de la Révolution. On note le paradoxe d'un discours qui célèbre l'héroïsme de l'armée tout en faisant l'éloge de la vertu et de la paix. La paix 
«ce mot si doux ! il verse à la fois dans l'âme et l'oubli des maux et les plus sincères espérances $\aleph^{35}$. Marengo suscite un élan d'enthousiasme, où les représentations des Lumières se mêlent à celles de la Révolution pour projeter tous les espoirs sur le nouveau régime. Dans un contexte propice aux célébrations, le sentiment patriotique génère l'illusion d'une re-fondation de la république dans la paix, avec ces mots magiques : concorde, paix, humanité, bonheur, patrie...

Les récits de presse traduisent l'éloquence vivante du spectacle et l'effet de tous les arts réunis pour illustrer la légende en marche de jeunes héros nimbés de gloire et d'immortalité poétique. Le Publiciste du 25 messidor rend compte de l'impression produite au Champ-de-Mars par la garde consulaire qui entourait Bonaparte: «ils étoient fatigués d'une longue route et ils sembloient sortir du champ de bataille. Cette espèce d'illusion ajoutoit encore à l'intérêt qu'ils inspirent. Ils ont reçu partout les témoignages de la reconnoissance nationale ». Bienaimé, dans La Clef du Cabinet, note l'émotion esthétique que suscite au Temple de Mars l'hymne de Méhul et Fontanes: "Le charme en était encore augmenté par l'exécution confiée aux citoyens Laïs, Chéron, Richer et Garat. On a remarqué sur-tout la strophe où les ombres des héros de la France, évoquées par le poëte, semblent venir dans le temple de Mars applaudir aux guerriers qui les ont suivi dans la carrière : elle était accompagnée d'une harpe et d'un cor, dont l'accord donne une idée complette de ce que les poëtes ont décrits comme les effets de l'harmonie céleste ».

31 Ce n'est pas assez de louer les belles actions, dit Despréaux dans son éloge, « il faut encore les investir de cet enthousiasme magique qui excite à les imiter». De l'art d'évoquer les mânes des héros dans les épisodes légendaires des guerres de la Révolution, aux récits qui décrivent les jouissances « d'un peuple libre, tout-à-la-fois acteur et spectateur de son triomphe », tout concourt à la représentation symbolique d'une communauté réconciliée autour de la figure héroïque du général-consul. Dans cette "représentation purement sensible de la liberté " (Kant), l'idéal habite les sommets emblématiques du passage des Alpes : s'interrogeant sur le consensus sublime réalisé par Bonaparte en messidor, le Journal des Hommes Libres expose comment ce guerrier pacificateur «nous a ramené tous, par nos intérêts les plus chers, autour de l'autel sacré de la patrie ». Les glaces du Mont-Saint-Bernard, offrent le symbole de ce ralliement autour du principe de tolérance universelle : « là, au-dessus de l'atmosphère des préjugés, plus près en quelque sorte du trône de l'éternel, on a vu la religion seconder le patriotisme, et des hospitaliers adorateurs de dieu, préparer le repas de nos soldats $»^{36}$.

\section{NOTES}

1.Jean-Claude Bonnet, Naissance du Panthéon : Essai sur le culte des grands hommes, Paris, Fayard, 1998, 419 p. 
2.L'article de Jaucourt dans l'Encyclopédie s'inspire de l'ouvrage de l'abbé Coyer, Dissertations pour être lues, la première sur le vieux mot de patrie, la seconde sur la nature du peuple, La Haye, Pierre Gosse Junior, 1755.

3.Bronislaw Baczko, Rousseau. Solitude et communauté, Paris, EPHE, 1974, pp. 312 et suivantes.

4.Albert Soboul, «Sentiment religieux et cultes populaires : saintes patriotes et martyrs de la liberté », Comprendre la Révolution, Paris, Maspéro, 1981, pp. 169-185. La mort de Marat, s.d. Jean-Claude Bonnet, Paris, Flammarion, 1986, 511 p. J'ai étudié la diffusion du culte à Paris dans Atlas de la Révolution française, 11, Paris, p. 83 (sous presse à l'EHESS), et « Mouvement patriotique et culte des martyrs de la liberté », Hommage à Michel Péronnet (à paraître).

5.Philippe Bordes, La Mort de Brutus de Pierre-Narcisse Guérin, publié à l'occasion de l'exposition Lucius Junius Brutus. L'Antiquité et la Révolution française, Musée de la Révolution française, Vizille, 1996, pp. 59 et suivantes.

6.Cuvres de Maximilien Robespierre, t. X, Paris, P.U.F., 1967, pp. 445, 459.

7.Philippe Goujard, "Une notion concept en construction : l'héroïsme révolutionnaire ", Dictionnaire des usages sociopolitiques (1770-1815), 2, Notions concepts, Paris, INALF, Klincksieck, 1987, pp. 9-44.

8.Le 30 prairial, une fête célèbre la liberté civile et religieuse au Temple de la Victoire ; le 11 messidor a lieu une messe solennelle à Saint-Laurent ( $L a$ Clef du Cabinet, $\mathrm{n}^{\circ} 1255$ ). 9.Journal des Défenseurs de la Patrie, $\mathrm{n}^{\circ}$ 1652. Le 20 messidor, les amis de la Religion naturelle célèbrent les victoires et rendent hommage à Desaix et à ses compagnons d'armes.

10.Éloge historique du général Desaix, par Joseph Lavallée, Paris, imp. des Sciences et des Arts, vendémiaire an IX, 1f, 53 p. Lavallée (marquis de Boisrobert, 1747-1816) avait déjà fait celui de Marceau en l'an VI.

11. $N^{\circ} 273$, 3 messidor ; l'article est reproduit dans Le Publiciste du 4. Un hommage de son compatriote Auzat est publié le 6 dans le Journal des Défenseurs de la Patrie.

12.Le Publiciste, 4 messidor.

13.Oraison funèbre du général Desaix..., par Destravault, Paris, chez l'auteur, 24 messidor an VIII, in $8^{\circ}, 23 \mathrm{p}$.

14.Journal des Défenseurs de la patrie, $n^{\circ} 1704,3$ fructidor, article extrait du Journal des Arts, signé Villeterque.

15.Précis de la vie. éloge funèbre du Général Desaix, par Simien Despreaux, Paris, floréal an IX, 59 p., seconde édition revue et augmentée, Paris, 1810. Sous la Restauration il est l'auteur d'un Louis XVII (1817) et des « Annales historiques de la Maison de France ». 16.Sur les éloges de Catinat, proposés pour le prix d'éloquence de l'Académie en 1775, voir Jean-Claude Bonnet, « Les morts illustres. Oraison funèbre, éloge académique, nécrologie ", Les Lieux de mémoire, s.d. Pierre Nora, II La nation***, pp. 217-241 (p. 223). 17. La Clef du Cabinet, $\mathrm{n}^{\circ} 1248$.

18.Moniteur, t. XXIX, p. 694, allusion ironique au Te Deum à Notre-Dame.

19. La Clef du Cabinet, nos 1261, 1262, Journal des Défenseurs de la Patrie, $n^{\circ} 1663$, Journal des Hommes Libres, $\mathrm{n}^{\circ} 220$.

20.Le Citoyen français, $\mathrm{n}^{\circ} 314$.

21.Jean Marcel Humbert, « Entre mythe et archéologie : la fortune statuaire égyptisante de Desaix et Kléber ", Le Culte des grands hommes au XVIIIe siècle, s.d. J. Pigeaud et J.-P. Barbe, Paris, Nantes, CRINI, 1998, pp. 219-232. Les formes furent jugées trop massives, et la maquette détruite un peu plus tard (Journal des Débats, 25 brumaire an IX). 
22.La Décade, n³3, pp. 366-368. Voir Yves Hersant, «L'invention d'Ossian », Le Culte des grands hommes, op. cit, pp. 103-107.

23. Le Publiciste, 20 prairial.

24.Journal des Hommes Libres, $\mathrm{n}^{\circ} 212,10$ messidor.

25.La Décade, $\mathrm{n}^{\circ}$ 33, 30 thermidor, p. 383.

26.Journal des Défenseurs de la Patrie, $n^{\circ} 1645,4$ messidor.

27.Le Publiciste, 3 messidor ; Journal de Paris, $\mathrm{n}^{\circ} 273$; La Clef du Cabinet, $\mathrm{n}^{\circ} 1249$; Journal des Hommes Libres, $\mathrm{n}^{\circ} 207$.

28. Le Publiciste, 14 messidor.

29.Journal des Défenseurs de la Patrie, $\mathrm{n}^{\circ}$ 1656, 15 messidor.

30.Journal des Hommes Libres, nos 216, 219 ; La Clef du Cabinet, $n^{\circ} 1262$.

31.Rapport du ministre de l'Intérieur aux Consuls, 23 prairial (La Clef du Cabinet, $\mathrm{n}^{\circ}$

1242).

32.La Clef du Cabinet, $\mathrm{n}^{\circ} 1271$; Journal des Défenseurs de la Patrie, $\mathrm{n}^{\circ} 1667$.

33.Journal de Paris, $\mathrm{n}^{\circ} 297$; Journal des Hommes Libres, $\mathrm{n}^{\circ} 226$.

34.Journal des Défenseurs de la Patrie, $\mathrm{n}^{\circ} 1668$.

35.Journal des Hommes Libres, $\mathrm{n}^{\circ} 215,13$ messidor.

36. $\mathrm{N}^{\circ} 212,10$ messidor.

\section{RÉSUMÉS}

Après la victoire de Marengo, la presse rend compte des hommages à la mémoire de Desaix dans les institutions particulières : ils se mêlent aux honneurs officiels et au tribut de reconnaissance qu'offre Paris à une armée nimbée de gloire et d'immortalité. Dans un contexte propice aux célébrations de la victoire et à l'embaumement des héros, les récits de presse amplifient le processus de ralliement universel au régime, sur les images parallèles du militaire philosophe (Desaix) et du guerrier pacificateur (Bonaparte). De l'art d'évoquer les mânes dans les éloges de la vertu héroïque aux récits qui décrivent le concert de vœux et l'enthousiasme du peuple, de l'annonce de la nouvelle de Marengo à l'apothéose patriotique du 14 juillet, tout concourt à la représentation symbolique d'une communauté réconciliée autour de la figure héroïque du général-consul.

Antique Virtue and New Heroes : the Press Records Desaix's Death and a Legendary Battle. After the victory of Marengo, the press gave accounts of the tributes paid to Desaix in private institutions, mixing them with official honours and marks of gratitude offered by Paris to an army haloed with glory and immortality. In a context conducive to victory celebrations and heroworship, the press record amplified the process of universal rallying to the regime, portraying in parallel the philosopher soldier (Desaix) and the peacemaker warrior (Bonaparte). Be it the art of conjuring up the shades of heroes and singing their virtue, or narrating the chorus of good wishes and popular enthusiasm, or acclaiming the victory of Marengo and whipping up patriotic fervour on 14 July, everything contributed to the symbolic representation of a community reconciled around the heroic figure of the General turned Consul. 
INDEX

Mots-clés : patriotisme, Marengo, héroïsme, éloges, esprit public

\author{
AUTEUR
}

RAYMONDE MONNIER

CNRS, ENS de Saint-Cloud 